\title{
Die Volkszählung von 1939 in Deutschland und Österreich - ein Beitrag zum Holocaust?
}

\author{
Gudrun Exner \\ Institut für Demographie, Wien
}

\begin{abstract}
Zusammenfassung: Im wissenschaftlichen Diskurs in Deutschland wird in letzter Zeit auch ein möglicher Beitrag der amtlichen deutschen Bevölkerungsstatistik zur Vernichtung der Juden diskutiert. Dieser Vorwurf stützt sich auf die Tatsache, dass im Rahmen der Volkszählung von 1939, die auch in der damaligen „Ostmark“ stattfand, eine Sonderzählung der Juden und ,jüdischen Mischlinge“ nach den Kriterien der antisemitischen Nürnberger Rassengesetze durchgeführt wurde. Quellenstudien zeigen, dass eine Verwendung von Volkszählungsdaten für die Erstellung von Transportlisten im Bereich des Möglichen lag, aber nicht belegt und aus verschiedenen Gründen eher unwahrscheinlich ist. In diesem Artikel wird der Problembereich zum ersten Mal auch für Österreich untersucht.
\end{abstract}

\begin{abstract}
The possibility of a contribution of the German official statistics to the Holocaust has recently become an issue of scientific interest in Germany. This accusation against the German population statistics is based on the fact, that the population census of 1939 also contained a special enumeration of the Jews and the "Jews of mixed ancestry" according to the categories of the anti-Semitic Nuremberg Laws. Historical investigations show, that it was at least possible that census data were used for transport list - but that could not be proved yet and seems to be improbable because of several reasons. The issue shall be investigated also for Austria in this article, which has not been done until now.
\end{abstract}

Schlüsselwörter: Bevölkerungsstatistik, Volkszählung, Nationalsozialismus, Holocaust.

\section{Einleitung}

Der vorliegende Artikel knüpft an eine aktuelle Diskussion innerhalb der deutschen Bevölkerungsstatistik und Demographie an, die bisher Österreich aus ihrem Blickfeld ausklammerte, obwohl, wie im Folgenden gezeigt werden soll, ein Österreichbezug durchaus gegeben ist. Es geht um die prekäre Frage, ob die zweite Volkszählung im Dritten Reich, die am 17. Mai 1939 auch in der damaligen „Ostmark“ stattfand, Datengrundlagen für die Deportationen der Juden und ,jüdischen Mischlinge“ bereitstellte.

Götz Aly und Karl-Heinz Roth stellten in ihrem 1984 erstmals erschienenen Buch „Die restlose Erfassung. Volkszählen, Identifizieren, Aussondern im Nationalsozialis- 
mus“ den Verdacht in den Raum, die Volkszählung von 1939 habe Daten für die Deportationslisten geliefert, ohne das allerdings so explizit und eindeutig zu formulieren (vgl. Aly/Roth, 2000, S. 93-95). Dieser Vorwurf basiert auf dem Faktum, dass der Zensus von 1939 auch eine Sonderzählung der Juden und ,jüdischen Mischlinge ersten und zweiten Grades“ im Sinne der Nürnberger Gesetze, also nach „rassischen“ Kriterien, beinhaltete. Dies war die zweite Sondererfassung der Juden in Deutschland nach der Zählung auf Grund des religiösen Bekenntnisses im Jahr 1933.

\section{Die deutsche Volkszählungsdebatte von 1983}

Das Erscheinen des Buches von Aly und Roth ist jedoch nicht nur im Kontext der historischen Forschung, sondern auch im Zusammenhang mit der Volkszählungsdebatte von 1983 in Deutschland zu sehen (vgl. Aly/Roth, 2000, S. 7).

Mit Stichtag vom 27. April 1983 war in der gesamten Bundesrepublik Deutschland die Durchführung einer Volks-, Wohnungs- und Arbeitsstättenzählung vorgesehen (vgl. Wimmer, 1983, S. 376). Doch das Vorhaben scheiterte. Zirka zwei Monate vor dem geplanten Termin begann sich eine Boykottbewegung zu formieren, die von einigen lokalen Bürgerinitiativen ihren Ausgang nahm und schließlich breite Teile der Bevölkerung erfasste. Da eine große Zahl von Bürgern den Absichten der Volkszählung schließlich misstraute, war nicht mit statistisch verwertbaren Ergebnissen der insgesamt 371 Millionen Mark teuren Zählung zu rechnen. Auf Grund von Unstimmigkeiten im Volkszählungsgesetz beschloss das Bundesverfassungsgericht Anfang April eine Aussetzung der Durchführung und eine Anpassung des Volkszählungsgesetzes an die neuere Entwicklung in der Datenverarbeitung. Rund 100 Millionen Mark Vorbereitungskosten waren damit verloren.

Vor allem folgende Punkte gaben zu berechtigter Kritik an der Zählung Anlass: Einerseits sollte der Zensus statistischen Zwecken und Planungsaufgaben dienen. Wohnverhältnisse, Beruf und Arbeitsstätte sollten erfasst werden. Genaue Daten hätte man hier aber nur bei Wahrung der Anonymität erhalten. Andererseits war ein Abgleich mit den Melderegistern vorgesehen, um eine geschätzte Zahl von zirka einer Million „Karteileichen“ seit der letzten Volkszählung im Jahr 1970 zu entfernen. Hierbei wurden aber die Volkszählungsdaten mit Name und Anschrift weitergegeben, die Anonymität also nicht gewahrt. Beide Ziele waren für sich genommen legitim und sinnvoll, in der Kombination aber unvereinbar (vgl. Der Spiegel, 1983, 13, S. 30).

Vor allem die vorgesehene Weitergabe der Daten an die Meldebehören - und damit an die Sicherheitsbehörden - sowie die Möglichkeit der Weitergabe an Länder und Gemeinden sowie private Interessensgruppen wie z.B. Hausbesitzer und kommerzielle Firmen ließen befürchten, dass diese Informationen zu Lasten der Auskunftspflichtigen verwendet würden. So befürchtete man, dass die Volkszählungsdaten von den Meldeämtern zur Herstellung von Personenkennzeichen, zur Zusammenführung von Daten und zur Erstellung von Persönlichkeitsprofilen verwendet würden, und dass Daten nach dem Prinzip der Rasterfahndung zur Ausfindigmachung von Einzelnen verwendet würden. Um ein konkretes Beispiel zu bringen: Wehrdienstpflichtige, die sich eine Wohnung in Berlin gemietet hatten, um sich befreien zu lassen, aber beispielsweise in Hamburg studierten oder arbeiteten, wären bei der Volkszählung 
„aufgeflogen“. Aber auch die Weitergaben von Daten an Gemeinden, insbesondere kleine Gemeinden, ließen Befürchtungen über einen Missbrauch aufkommen (vgl. Der Spiegel, 1983, 13, S. 30-32).

Mit Sicherheit wies das Volkszählungsgesetz rechtliche Mängel auf. Nach Ansicht von amtlichen Datenschutzbeauftragten lag ein Grund dafür sicher auch in der Tatsache, dass die technische Entwicklung sich seit der letzten deutschen Volkszählung von 1970 auf dem Gebiet der Datenverarbeitung stark beschleunigt hatte und dass die Gesetzgebung mit dieser Entwicklung nicht Schritt gehalten hatte (vgl. Der Spiegel, 1983, 12, S. 110).

Die Diskussion der Sachverhalte wurde von einer politisch-ideologischen Debatte überlagert. Konservative Kreise, etwa auch die „Frankfurter Allgemeine“, werteten den Volkszählungsboykott in stereotyper Form als einen Angriff auf das ganze System, auf den Rechtstaat und sahen im Verfassungsgerichtsurteil eine „Niederlage des Staates“ (vgl. Fromme, 1983, S. 1). Der „Spiegel“ dagegen berichtete eher aus der Perspektive der Kritiker des Zensus und begrüßte die Entscheidung des Bundesverfassungsgerichtes unter Präsident Ernst Benda als ein Zeichen, dass das staatliche System zur Selbstbesinnung und sachlichen Korrektur fähig sei (vgl. Der Spiegel, 1983, 16, S. 19 f.).

Die Diskussion um die Volkszählung von 1983 in Deutschland wurde jedoch fast ausschließlich gegenwartsbezogen geführt - von vereinzelten Hinweisen auf die „Datengier“ des Dritten Reichs abgesehen. Die Debatte griff nicht auf Österreich über hier hatte die Volkszählung schon 1981 stattgefunden. Für Historiker wie Aly und Roth wurde die Volkszählungsdebatte jedoch zum Anlass, die Vergangenheit quasi aus vergleichender Perspektive zu untersuchen, insbesondere der Frage nach einer Weitergabe der Daten des Zensus von 1939 an die polizeilichen Meldestellen anhand der Quellen nachzugehen.

\section{Die Volkszählung von 1939 in der historischen For- schung}

Der von vielen Forschern, aber von Aly und Roth bisher am plakativsten formulierte Vorwurf gegen die deutsche amtliche Bevölkerungsstatistik bedurfte einer Klärung durch ein umfassendes Studium der zu diesem Problembereich vorliegenden Quellen. Eine solche Studie wurde von der Historikerin Jutta Wietog mit Unterstützung des deutschen Statistischen Bundesamts im Jahr 2001 vorgelegt. Wietogs Arbeit „Volkszählungen unter dem Nationalsozialismus: eine Dokumentation zur Bevölkerungsstatistik im Dritten Reich" stellt eine umfassende Quellenstudie dar. Wietog zeigt auf, dass die Vorgänge rund um den Zensus von 1939 komplizierter und komplexer waren, als Aly und Roth dies darstellen. Sie bestätigt aber letztlich die Ergebnisse von Aly und Roth, dass nämlich Volkszählungsdaten tatsächlich - auf dem Umweg über die Melderegister und die so genannte „Volkskartei“ (eine Kartei zur Ergänzung der Melderegister) - in die Hände der Gestapo gelangen konnten (vgl. Wietog, 2001, S. 168), welche die Deportationen in die Vernichtungslager durchführte. Dafür gibt es jedoch keinen expliziten Beleg. Denn Karteikarten und Deportationslisten 
enthalten üblicherweise keine Vermerke, woher die Daten stammen. Hier ist man auf den innerbehördlichen Schriftverkehr angewiesen. Wietog schwächt den obigen Befund aber mit der Feststellung ab, dass die Gestapo und SS viele Quellen hatten, welchen sie Daten für Deportationslisten entnehmen konnten. Vor allem wurden die jüdischen Kultusgemeinden gezwungen, ihre Mitglieder zu registrieren und diese Daten an die NS-Stellen weiterzuleiten (vgl. Wietog, 2001, S. 74 f.).

Eine Reihe von weiteren Gründen schränken die Möglichkeit, dass Zensusdaten missbraucht wurden, ebenfalls ein. Zum einen sah das Volkszählungsgesetz für den Zensus von 1939 in seinen verschiedenen Versionen vor, dass die Volkszählungsdaten nur vom Statistischen Reichsamt bzw. den selbstaufbereitenden Statistischen Landesämtern ausgewertet werden durften. Diese Bestimmung galt auch für Österreich. Sonderauszählungen vor dieser gesamtstaatlichen Auswertung, etwa durch Gemeinden, waren ausdrücklich untersagt (vgl. Gesetzblatt 1939, Nr. 362, § 4 (3)). Das lässt den Schluss zu, dass es für die NS-Stellen keine legale Möglichkeit gab, vor Abschluss der zentralen Auswertung an die Daten heranzukommen, obwohl der Wunsch nach dem sofortigen Zugriff auf die Daten durchaus bestand. Wietog bemerkt hierzu, dass die Daten aus der Judenerfassung den Meldeämtern erst im Frühjahr 1941 zur Verfügung gestellt wurden (vgl. Wietog, 2001, S. 191). Da die Massentransporte jedoch im Herbst 1941 einsetzten, wäre genug Zeit für einen Datentransfer geblieben. Allerdings konnten die Daten, beispielsweise zur Wohnadresse, zu diesem Zeitpunkt bereits überholt sein. Die Sondererfassung der Juden wurde mit Hilfe eines eigenen Fragebogens durchgeführt, der in einem verschlossenen Umschlag an das Zählorgan abgegeben werden sollte (vgl. Gesetzblatt 1938, Nr. 336, S. 1449). Auch dadurch sollte die Geheimhaltungspflicht auf unterster Ebene gewahrt werden. Aber der Fragebogen enthielt alle Daten, die nötig waren, um die Deportationen durchzuführen insbesondere den vollen Namen, das Geburtsdatum und die Wohnadresse.

\section{Die Durchführung der Volkszählung von 1939 in Österreich}

Von deutscher Seite, aber auch von der österreichischen historischen Forschung wurden die Hintergründe des Zensus von 1939 in Österreich noch nicht untersucht. Die in jüngster Zeit von d. Verf. durchgeführten Recherchen auf diesem Gebiet brachten manches interessante Detail zu Tage. Mit dem Anschluss im März 1938 verlor das österreichische Bundesamt für Statistik seine Selbständigkeit und wurde zunächst dem Statistischen Reichsamt unterstellt. Der letzte Präsident, Karl Karwinsky-Karwin (18881957), wurde nach Dachau verschleppt (vgl. Zeller, S. 280). Wilhelm Winkler (18841984), der bisherige Abteilungsleiter für Bevölkerungsstatistik, der die österreichische Volkszählung von 1934 in ausgezeichneter Weise durchgeführt hatte, wurde zwangsweise in den Ruhestand versetzt, da er mit einer Jüdin verheiratet war und nicht zu einer Scheidung überredet werden konnte (vgl. Pinwinkler, 2001, II, S. 34). Neuer Vorstand der nunmehr als „Österreichisches Statistisches Landesamt" bezeichneten Institution wurde Felix Klezl, Freiherr von Norberg (1885-1972) als Vizepräsident. Neuer Abteilungsleiter für Bevölkerungsstatistik wurde ein bisheriger Mitarbeiter von 
Winkler, Oskar Gelinek (1910-1944) (vgl. Lebmann/Helczmanovszki, 1986, S. 50). Unter Klezl und Gelinek wurde die Volkszählung von 1939 auch in Österreich durchgeführt. Es erschien keine selbständige Publikation der Volkszählungsergebnisse durch das Österreichische Statistische Landesamt. Die Volkszählungsdaten wurden gemeinsam mit den deutschen Ergebnissen in der Reihe „Statistik des Deutschen Reichs“, Bd. 552, Heft 1-5 publiziert. Klezl war kein NSDAP-Mitglied (vgl. Gauakt Klezl-Norberg) und wurde nach der Volkszählung durch den „zuverlässigeren“, aber weniger bedeutenden Parteigenossen Friedrich Hribar ersetzt. Das Amt wurde unter Hribar der Behörde des „Reichsstatthalters Wien“ unterstellt (vgl. Gauakt Hribar) und dürfte in seiner Tätigkeit noch weiter eingeschränkt worden sein. Hribar scheint in der Literatur über die Geschichte der österreichischen zentralen amtlichen Statistik übrigens nicht auf, erst ein Gauakt über Felix Klezl gab den entscheidenden Hinweis. In den Unterlagen zu Hribar wird dieser als umgänglicher, aber durchschnittlicher Charakter beschrieben. Materialen aus der Zeit nach dem Krieg enthalten Entlastungsschriften von ehemaligen Mitarbeitern und Mitarbeiterinnen, die Hribar trotz deren ihm bekannter Ablehnung des Regimes nicht denunziert hatte (vgl. Liquidator).

Die Begleitumstände der Deportationen in Wien lassen den Schluss zu, dass die Volkszählung in Österreich hierbei keine zentrale Rolle spielte. Über 90 Prozent der österreichischen Juden befanden sich zur Zeit des Anschlusses in Wien (vgl. Moser, 1999, S. 16). Nach der Machtübernahme kam es zu massenhaften Vertreibungen aus den Wohnungen. Die Wiener Juden wurden in Wohnungen im II., IX. und XX. Bezirk „umgesiedelt“ und hier in bestimmten Gassen konzentriert (vgl. Botz, 1975, S. 73 f., S. 78). Die Gestapo wusste, in welchen Stadtbereichen sich die jüdischen Bürger aufhielten. Es hätte also genügt, einfach diese Gassen zu durchkämmen. Die Juden waren ja an dem gelben Stern sofort zu erkennen. Der Abtransport in die Sammellager, welche die erste Stufe der Deportationen darstellten, erfolgte jedoch aufgrund von Namens- und Adressenlisten. Die Adressen müssen allerdings nicht den Zensusdaten entnommen worden sein, waren doch auf Grund der „Umsiedlungen“ viele Wohnungsanschriften nicht mehr gültig. Möglicherweise stammten die Daten hierzu aus der groß angelegten Zählung, die im September 1939 - vier Monate nach der Volkszählung - von der Israelitischen Kultusgemeinde Wien unter den Wiener Glaubensjuden vorgenommen wurde. Die IKG befand sich damals bereits völlig in der Hand von Adolf Eichmann, und Historiker meinen, dass er diese Zählung angeordnet hat, wenn es dafür auch keinen expliziten Beleg gibt (vgl. Rosenkranz, 1978, S. 213). Die Wiener Glaubensjuden wurden über die Zeitschrift der IKG, das ,Jüdische Nachrichtenblatt" aufgefordert, in der Zeit vom 10. bis 20. September 1939, je nach Anfangsbuchstabe des Familiennamens zu einem bestimmten Tag, bei der IKG zu erscheinen und den Meldezettel mitzubringen (vgl. Jüdisches Nachrichtenblatt 1939, $71 / 72$ S. 1). Es ist mit großer Wahrscheinlichkeit anzunehmen, dass die Adresse registriert wurde. Damit wäre die wichtigste Datengrundlage für die Deportationen bei dieser Zählung erhoben worden. Da die meisten Juden Wiens zudem der Jüdischen Kultusgemeinde angehörten, wäre mit dieser Zählung tatsächlich der Großteil der Wiener und damit der österreichischen Juden erfasst worden. Bei der Zählung vom 10. bis 20. September 1939 wurden 65.822 Juden registriert (vgl. Jüdisches Nachrichtenblatt vom 20. November 1939, S. 1). 


\section{Einige Bemerkungen zur Quellenlage}

Das vorliegende Quellenmaterial und die Sonderzählung der Wiener Glaubensjuden im September 1939 unterstützen nicht gerade die Hypothese, dass in Österreich Volkszählungsdaten für die Transportlisten herangezogen wurden. Hier muss man aber eines berücksichtigen: Viel Quellenmaterial wurde vernichtet oder durch Kriegseinwirkungen zerstört. So ist etwa laut Auskunft des Wiener Stadt- und Landesarchivs der Schriftverkehr rund ums Wiener Meldewesen nicht mehr erhalten. (Wir erinnern uns: Volkszählungsdaten sollen über die Melderegister in die Hände der Gestapo gelangt sein.) Auch viele Gestapoakten wurden vernichtet. Vielleicht kann eine nochmalige minutiöse Suche in den Beständen des Österreichischen Staatsarchivs Hinweise zu Tage fördern, aber besonders wahrscheinlich ist dies nicht. Aussagen von Zeitzeugen können als Ergänzungen herangezogen werden. Hier ergab sich aber, dass viele „Insider" von damals bereits verstorben sind, oder dass ihre Aussagen mit Vorbehalten zu betrachten sind. So teilte mir ein ehemaliger leitender Beamter des Österreichischen Statistischen Zentralamts mit, als er 1957 ins Amt eingetreten sei, habe er vom Gerücht gehört, dass anlässlich des Zensus von 1939 Studenten angestellt wurden, welche die Daten der Juden in lange Listen einzutragen hatten (vgl. Interview Lenhart, S. 1). Nach dem Volkszählungsgesetz wäre ein solches Vorgehen in legaler Weise nicht möglich gewesen (siehe oben). Aussagen dieser Art können daher nur als weiterführender Hinweis betrachtet werden.

\section{Resümee}

Eine direkte Beteiligung auch der österreichischen zentralen amtlichen Statistik an der Durchführung der Judenvernichtung kann daher wie im Fall Deutschlands weder eindeutig belegt noch eindeutig ausgeschlossen werden. Fest steht aber, dass die zentrale amtliche Statistik in Deutschland und Österreich wie viele andere staatliche Ämter eine willige Helferin des NS-Regimes wurde und, wie zahlreiche Untersuchungen belegen, die NS-Bevölkerungs- und Rassenpolitik unterstützte.

\section{Literatur}

„An die in Wien wohnenden Juden! ...“. In: Jüdisches Nachrichtenblatt. Ausgabe Wien, 71/72: 1, 1939.

„Beten, daß die Sicherungen halten“. Rechtsinformatiker Wilhelm Steinmüller über die geplante Volkszählung. In: Der Spiegel, 12: 106-110, 1983.

„Laßt 1000 Fragebogen glühen“. In: Der Spiegel, 13: 28-32, 1983.

„Ohne Drohgebärde, ohne Angst“. In: Der Spiegel, 16: 17-23, 1983. 
G. Aly and K.H. Roth. Die restlose Erfassung. Volkszählen, Identifizieren, Aussondern im Nationalsozialismus. Fischer Taschenbuch, Berlin, $2^{\text {nd }}$ edition, 2000.

G. Botz. Wohnungspolitik und Judendeportation in Wien 1938 bis 1945. Zur Funktion des Antisemitismus als Ersatz nationalsozialistischer Sozialpolitik. Geyer, Wien, Salzburg, 1975.

F. K. Fromme. Ein Sieg über den Staat. In: Frankfurter Allgemeine, 86/15 D: 1, 1983.

Jüdisches Nachrichtenblatt. Ausgabe Wien, vom 20. November 1939.

R. Lebmann and H. Helczmanovszki. Auf dem Gebiete der Bevölkerungsstatistik und Bevölkerungswissenschaft tätige Österreicher. Eine Biographie und Bibliographie. Österreichische Akademie der Wissenschaften, Wien, 1986.

J. Moser. Demographie der jüdischen Bevölkerung Österreichs. Dokumentationsarchiv des Österreichischen Widerstandes, Wien, 1999.

A. Pinwinkler. Wilhelm Winkler (1884-1984) - eine Biographie. Ein Beitrag zur Geschichte der Statistik der Demographie in Österreich und Deutschland. Phil. Diss., Salzburg, 2001.

H. Rosenkranz. Verfolgung und Selbstbehauptung. Die Juden in Österreich 1938-1945. Herold, Wien, München, 1978.

J. Wietog, Volkszählungen unter dem Nationalsozialismus: eine Dokumentation zur Bevölkerungsstatistik im Dritten Reich. Duncker \& Humboldt, Berlin, 2001.

S. Wimmer. Volks-, Wohnungs- und Arbeitsstättenzählung 1983. In: Der Städtetag, 6: 376-380, 1982.

W. Zeller. Geschichte der zentralen amtlichen Statistik in Österreich, Anhang I. Die Leiter der österreichischen Verwaltungsstatistik. In: Österreichisches Statistisches Zentralamt, editor, Geschichte und Ergebnisse der zentralen amtlichen Statistik in Österreich 1829-1979. Festschrift aus Anlaß des 150jährigen Bestehens der zentralen amtlichen Statistik in Österreich, pages 263-283. Österreichische Staatsdruckerei, Wien, 1979.

\section{Quellen}

Gesetzblatt für das Land Österreich. 1938. Nr. 336, S. 1443-1524.

Gesetzblatt für das Land Österreich. 1939. Nr. 362, S. 1248-1250.

Interview mit Viktor Lenhart vom 20. März 2002, Protokoll. 
Österreichisches Staatsarchiv (ÖStA) / Archiv der Republik (AdR) / Gauakt Friedrich Hribar.

ÖStA / AdR / Gauakt Felix Klezl-Norberg

ÖStA / AdR / BKA / Liquidator / R.Sth. Wien 2 / GZ. 4568/47.

Adresse der Autorin:

Dr. Gudrun Exner, Historikerin Laimgrubeng. $7 / 8$

A-1060 Wien

Austria

E-mail: gudrun.exner@utanet.at 